\title{
Response to comments on Maffulli and Del Buono: Release of the medial head of the gastrocnemius for Achilles tendinopathy in sedentary patients
}

\author{
Nicola Maffulli $^{1,2}$ • Angelo Del Buono ${ }^{3}$
}

Received: 28 February 2015 / Accepted: 1 March 2015 /Published online: 19 March 2015

(C) SICOT aisbl 2015

Dear Editor,

Many thanks for allowing us to examine the comments by Zang et al. on our recent article, "Release of the medial head of the gastrocnemius for Achilles tendinopathy in sedentary patients: a retrospective study" [1], and answer the issues they have raised.

We respectfully point out that some of the points raised by the authors of the letter are truisms that do little to advance the science and understanding of the condition at hand. Nevertheless, we shall try and answer each point raised in the order in which they were posed:

1. This suggestion is contradictory. Zhang et al. suggest that the sample size is small. Indeed, this is what we ourselves acknowledged and expressly considered a limitation of the study. Given the sample size, the descriptive statistics and the analysis reported in the article are more than adequate.

Nicola Maffulli

n.maffulli@qmul.ac.uk

Angelo Del Buono

angelo_delbuono@libero.it

1 Centre for Sports and Exercise Medicine, Barts and The London School of Medicine and Dentistry, Mile End Hospital, 275 Bancroft Road, London E1 4DG, England, UK

2 Department of Musculoskeletal Disorders, Faculty of Medicine and Surgery, University of Salerno, Salerno, Italy

3 Department of Orthopaedic and Trauma Surgery Hospital Sant' Anna, 22020 Via Ravona, Como, Italy
2. Zhang et al. contradict themselves. On the one hand, they suggest that the overall sample is small (an issue that we ourselves had expressly outlined in the original manuscript) and, on the other hand, they suggest that we should carry out subgroup analyses on the various subsamples according to the previous management that the patients received. Given the small numbers, this is a moot point.

3. Please see above.

4. We acknowledge that we did not perform a healthcare cost analysis, and that costs can, and do, vary according to the various healthcare systems, even in the same country. Obviously, we cannot avoid the risk that readers overinterpret what an author writes. It appears that this is what has happened in this instance. In regards to the issue about calf measurements and strength, we respectfully point out that we expressly stated that these patients were not athletic. Therefore, in such patients the issue of strength is less important than in athletic patients. We also respectfully point out that the indication for surgery in Achilles tendinopathy is pain. Finally, we stress that there is a profound philosophical difference between statistically significant and clinically relevant. In this instance, as obvious in the text, a statistically significant difference did not translate into a clinically relevant effect.

5. This point is a truism that does not need further comment.

\section{Reference}

1. Maffulli N, Del Buono A (2015) Release of the medial head of the gastrocnemius for Achilles tendinopathy in sedentary patients: a retrospective study. Int Orthop 39(1):61-65. doi:10.1007/s00264-0142553-1 